\title{
Delay in achieving enteral autonomy and growth outcomes in very low birth weight infants with surgical necrotizing enterocolitis
}

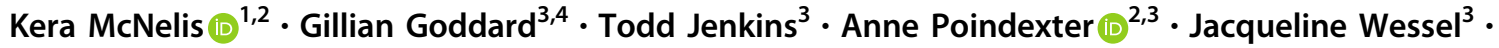 \\ Michael Helmrath ${ }^{2,3} \cdot$ Brenda Poindexter ${ }^{1,5}$
}

Received: 2 September 2020 / Revised: 26 October 2020 / Accepted: 13 November 2020 / Published online: 2 December 2020

(c) The Author(s), under exclusive licence to Springer Nature America, Inc. 2020

\begin{abstract}
Objective To understand the nutritional intake and growth outcomes of very low birth weight infants with surgical necrotizing enterocolitis (NEC).

Study design In a retrospective cohort study, linear mixed models were used to compare growth outcomes from birth to 24 months corrected age for very low birth weight (VLBW) infants with surgical NEC to those with spontaneous intestinal perforation (SIP). Kaplan-Meier curves were developed to demonstrate the duration of parenteral nutrition (PN) use.

Result Height differed by surgical NEC and SIP over time (interaction $p=0.03$ ). Surviving infants with surgical NEC had lower head circumference z-scores at 24 months. Of infants surviving surgical NEC, 71\% received PN for $>60$ days after diagnosis.

Conclusion The majority of infants with surgical NEC have a delay in achieving enteral autonomy. There was a difference in linear catch-up growth over time between infants with SIP and surgical NEC at 24 months.
\end{abstract}

\section{Introduction}

Growth failure in preterm infants following necrotizing enterocolitis (NEC) is multifactorial. Inability to provide enteral feeding during the acute phase of the disease, incomplete bowel length following surgical resection, malabsorption, and complications like obstruction or stricture are all risk factors for growth failure even with use of parenteral nutrition (PN). Infants with severe NEC are at risk for intestinal failure, or the inadequate

Kera McNelis

Kera.mcnelis@cchmc.org

1 Division of Neonatology, Cincinnati Children's Hospital Medical Center, Cincinnati, OH, USA

2 University of Cincinnati, College of Medicine, Cincinnati, $\mathrm{OH}$, USA

3 Division of Pediatric and General and Thoracic Surgery, Cincinnati Children's Hospital Medical Center, Cincinnati, $\mathrm{OH}$, USA

4 Department of Surgery, Boston Medical Center, Boston, MA, USA

5 Department of Pediatrics, Emory University, Atlanta, GA, USA intestinal absorption of nutrients that leads to growth failure and dependence on PN [1]. Optimizing growth in these infants can improve neurodevelopmental outcomes [2]. It is known that higher parenteral protein provision following surgery for NEC leads to improved head circumference growth [3]. Understanding which of these factors are modifiable will assist care providers in focusing resources.

To better understand the growth outcomes for preterm infants with NEC, we studied very low birth weight (VLBW) infants with surgical NEC and compared their management and long term growth outcomes to those with spontaneous intestinal perforation (SIP). VLBW infants with SIP were chosen as the control as they were expected to have comparable average birth weights and gestational ages. Additionally, both groups experience a surgical procedure and are expected to receive similar management by being cared for by the same group of surgeons and neonatologists (e.g., ventilator support, antibiotic use, etc.). These groups might have different functional ability to achieve enteral autonomy, or independence from PN [4]. We hypothesized that infants with surgical NEC would have restricted growth in comparison to infants with SIP, and that this would be attributable to differences in nutritional provision. 


\section{Subjects and methods}

This retrospective study included all infants admitted to a Level IV, referral neonatal intensive care unit (NICU) with dates of birth between 1/1/2011 and 12/31/2016 with a diagnosis of either NEC or SIP. This institution provides consistent nutritional management for such patients with oversight by a dedicated NICU intestinal rehabilitation rounding team. The Institutional Review Board at Cincinnati Children's Hospital Medical Center (CCHMC) approved this study prior to initiation of study procedures (IRB\# 9223). The list of potentially eligible subjects was generated through billing codes in the electronic medical record (Fig. 1). This data set was also used in a separate analysis; details of classification of the infants is described elsewhere [5]. Manual chart review of clinical and surgical data including progress notes, surgical reports, and pathology reports, was accomplished through agreement by two study investigators to verify diagnosis and determine study inclusion. A third investigator adjudicated in case of dissent. Subjects were excluded if they did not meet diagnostic criteria for either SIP or NEC, if they had a birth weight greater than $1500 \mathrm{~g}$, if they had a diagnosis of both SIP and NEC, or if NEC or SIP was a historical diagnosis and infant did not receive care for the disease during the admission at the study institution. Infants were excluded if they had a congenital gastrointestinal anomaly such as gastroschisis. NEC was classified by the modified Bell's staging criteria, and infants were designated "surgical NEC" if they received a peritoneal drain or laparotomy for treatment of NEC $[6,7]$. Study data were extracted from the electronic medical record, and then collected and managed using Research
Electronic Data Capture (REDCap) tools hosted at CCHMC [8].

Extracted data included demographic and baseline characteristics and surgical interventions. Details of enteral feeding, parenteral nutrition provision, and growth were included at multiple time points through initial hospitalization and follow up visits through 24 months corrected age. Subjects were considered to have achieved enteral autonomy if their growth and nutrient accretion was successful after discontinuation of PN per their clinician. Complete anthropometric measurements were trended weekly during hospitalization and during appointments in the neonatal high risk follow up clinic after discharge. In the follow up clinic, recumbent length was measured with length boards and a single examiner. Head circumference was measured with a non-stretch tape. Anthropometric $z$ scores were derived from the Fenton growth chart through 50 weeks corrected age and World Health Organization growth charts thereafter $[9,10]$.

Data were extracted from surgical notes. The actual remaining small bowel length was directly measured by the surgeon during the operation. Expected bowel length values were generated per corrected age, weight, and height for the infant on the day of surgery using Struijs' normative reference data [11], and these three values were averaged. The percent of remaining small bowel length was then calculated by dividing the directly measured actual remaining bowel length by the expected normative intestinal length per Struijs.'

Statistical software JMP Pro Version 14.0 (SAS institute, Cary, North Carolina) was used for analysis of de-identified data. Comparison of categorical demographic and outcome
Fig. 1 Flow diagram of study subject selection. Potentially eligible subjects were generated through billing codes. Manual chart review was completed with exclusion of ineligible infants.

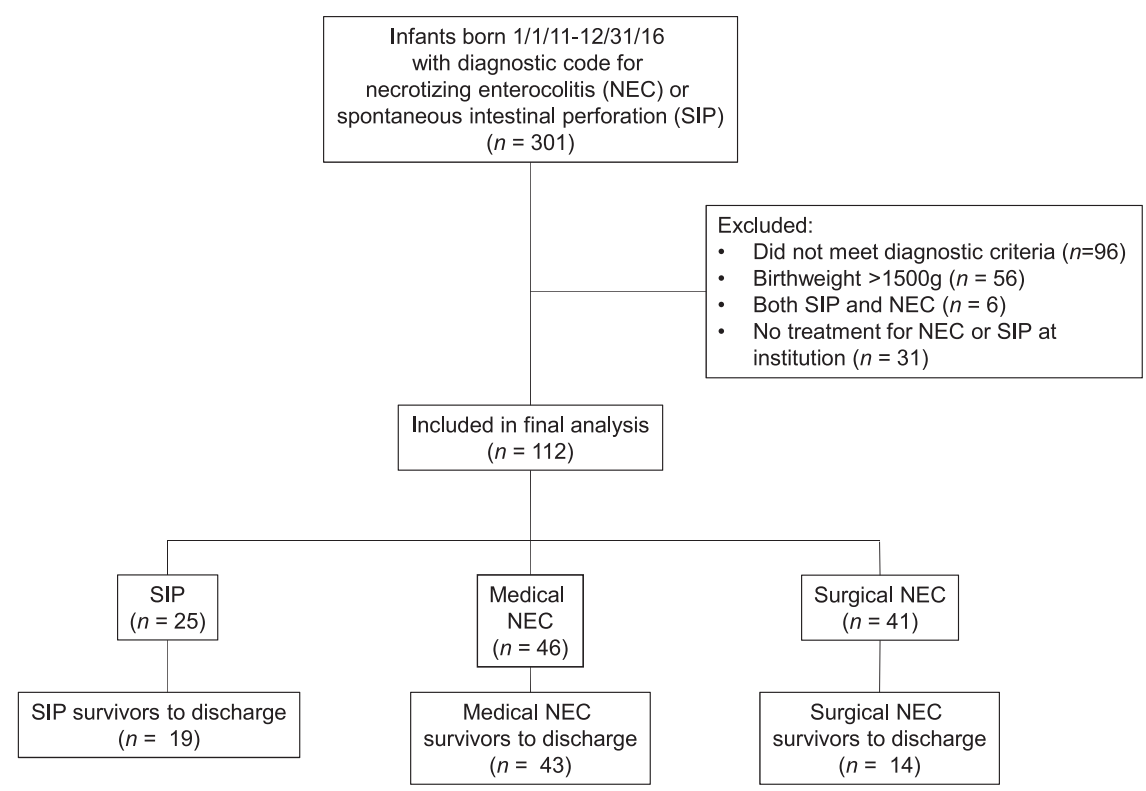


characteristics of surviving infants with SIP and surgical NEC was made with Fisher's exact tests. Wilcoxon ranksum test were used for group comparisons of continuous variables. Log-rank tests from Kaplan-Meier analyses were used to compare the duration of $\mathrm{PN}$ for surviving infants with medical NEC, surgical NEC, and SIP. Linear mixed models were used to compare longitudinal growth outcomes (weight, height, head circumference) by NEC and SIP groupings. The following variables were considered for inclusion in each model: NEC/SIP status, study visit, NEC/ SIP by visit interaction, gestational age, and sex. Modeled means and $95 \%$ confidence intervals were calculated by NEC/SIP status and study visit. All significance tests were two-sided with a critical alpha level of 0.05 .

\section{Results}

Three hundred and one unique infants were identified as potentially eligible through electronic medical record extraction. Of these, 112 met inclusion criteria (Fig. 1). Most of the infants who were excluded for birth weight $>1500 \mathrm{~g}$ had a congenital anomaly that posed additional risk for ischemic bowel disease, such as gastroschisis. The 46 infants with medical NEC were only included in the analysis of PN duration. Of the 25 VLBW infants with SIP, 19 survived to hospital discharge. Two infants with SIP died after initial hospitalization of unknown causes (unexpected deaths at home, one of these infants had a ventriculoperitoneal shunt), so that 17 infants were followed to 24 months corrected age. The final analysis included 41 VLBW infants with surgical NEC, of which 14 survived to hospital discharge. The majority $(73 \%)$ of infants with surgical NEC who did not survive to NICU discharge died within $72 \mathrm{~h}$ of diagnosis of NEC. One infant with NEC died unexpectedly after initial hospitalization at home; this infant had a tracheostomy tube.

Table 1 shows the baseline and outcome characteristics of the surviving VLBW infants with a history of SIP or surgical NEC. There were no statistically significant differences in the demographic characteristics of these infants. There were no statistically significant differences in comorbidities related to prematurity, with the exception of cholestasis. Other than complications related to prematurity, one of the infants with surgical NEC also had Tetralogy of Fallot, and one infant with SIP had a sacrococcygeal teratoma.

Infants with surgical NEC were discharged at a significantly later corrected age, and this was due to a longer length of stay and not an earlier gestational age at birth (Table 1). The median length of small bowel remaining in this group was $86.1 \%$ (interquartile range $39.9 \%-97.0 \%$ ). Only 1 of $14(7 \%)$ surviving VLBW infants with surgical NEC were diagnosed with NEC totalis at first laparotomy. Four of 14 (29\%) qualified for a diagnosis of short bowel syndrome using a definition of $<50 \%$ remaining short bowel.

Figure 2 displays the duration of PN provision for surviving VLBW infants with each stage of NEC and infants with SIP after the respective diagnosis was made. Surviving VLBW infants with surgical NEC had a median duration of 89 days (95\% CI: 29, 188), significantly longer than that for infants with medical NEC (29.5 days [95\% CI: 23, 43]; $p<0.001)$ and infants with SIP (35 days [95\% CI: 27, 53],
Table 1 Comparison of surviving infants with surgical necrotizing enterocolitis or spontaneous intestinal perforation.

\begin{tabular}{llll}
\hline & Surgical NEC, $n=14$ & SIP, $n=19$ & $p$ value \\
\hline Baseline characteristic & & & \\
Birth weight $(\mathrm{g})$ & $765(650-1030)$ & $855(732-1157)$ & 0.38 \\
Gestational age (weeks) & $25.9(24.7-27.7)$ & $26.6(25.3-28.6)$ & 0.43 \\
Gender, female & $57.1 \%(8)$ & $47.4 \%(9)$ & 0.73 \\
Antenatal steroids & $16.7 \%(2)$ & $15.8 \%(3)$ & 1.0 \\
Comorbidity & & & \\
Culture (+) sepsis & $57.1 \%(8)$ & $36.8 \%(7)$ & 0.30 \\
Bronchopulmonary dysplasia & $78.6 \%(11)$ & $63.2 \%(12)$ & 0.46 \\
Intraventricular hemorrhage, Surgical-4 & $21.4 \%(3)$ & $26.3 \%(5)$ & 1.0 \\
Patent ductus arteriosus & $71.4 \%(10)$ & $42.1 \%(8)$ & 0.16 \\
Retinopathy of prematurity requiring treatment & $21.4 \%(3)$ & $15.8 \%(3)$ & 1.0 \\
Cholestasis(Direct bilirubin $\geq 2)$ & $64.3 \%(9)$ & $26.3 \%(5)$ & 0.03 \\
Management & & & \\
Length of stay (days) & $180(108-269)$ & $101(77-135)$ & 0.01 \\
Corrected age at discharge (weeks) & $50.5(41.6-58.8)$ & $41.9(39-46.6)$ & 0.03 \\
Initial management: laparotomy & $78.6 \%(11)$ & $42.1 \%(8)$ & 0.04 \\
\hline Data
\end{tabular}

Data are median (interquartile range) or percent (number). 
$p=0.004)$. Duration between infants with medical NEC and SIP did not statistically differ $(p=0.27)$. The majority $(10 / 14,71 \%)$ of surviving VLBW infants with surgical NEC received PN for greater than 60 days. A substantial minority $(6 / 14,43 \%)$ of this group received $\mathrm{PN}$ for $>90$ days. Two of these infants remained on $\mathrm{PN}$ at a year of life.

Initial enteral feeding was maternal breast milk when available or supplemental pasteurized donor milk from the OhioHealth Mothers' Milk Bank (Columbus, OH). Infants were not discharged home with donor milk and were transitioned to a supplement formula prior to discharge (Table 2). Both groups received on average $24 \mathrm{kcal} / \mathrm{oz}$ enteral feeds at discharge. Three infants with surgical NEC were discharged to home with $\mathrm{PN}$, and these infants received each 45,51 , and $116 \mathrm{~mL} / \mathrm{kg}$ of enteral feeding volume in addition to PN. While only $10.5 \%$ of infants with SIP had a subsequent hospitalization related to bowel disease compared to $38.5 \%$ of infants with surgical NEC, this did not reach statistical significance $(p=0.09)$. There was not a statistically significant difference in the total number of gastroenterology, neonatal, and surgery follow up appointments in the first 24 months of corrected age between infants

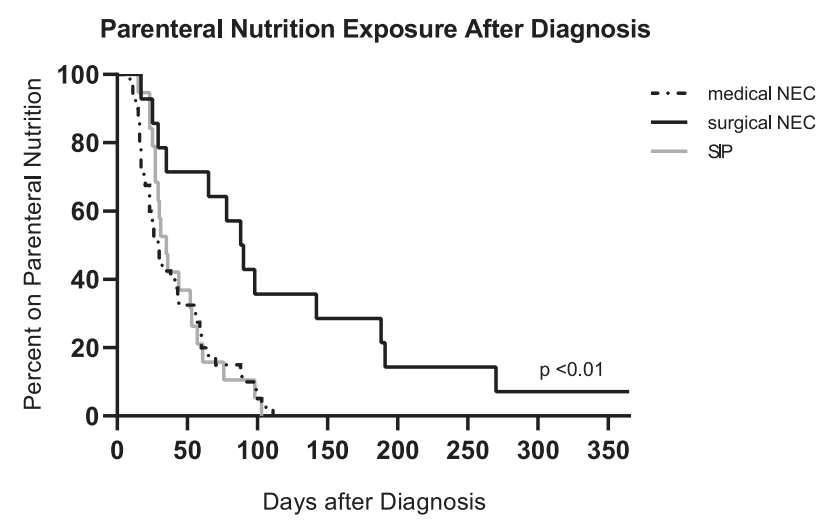

Fig. 2 Duration of parenteral nutrition exposure after diagnosis of necrotizing enterocolitis or spontaneous intestinal perforation. This Kaplan-Meier curve displays duration of parenteral nutrition exposure (days) in 19 very low birth weight (VLBW) infants diagnosed with spontaneous intestinal perforation, 43 VLBW infants with medical necrotizing enterocolitis (NEC), and $14 \mathrm{VLBW}$ infants with surgical NEC. Included infants survived to hospital discharge. with surgical NEC (median 9 appointments) and infants with SIP (median 6 appointments, $p=0.12$ ).

At 18-24 months corrected age, three surviving infants with SIP were missing anthropometric data and two surviving infants with surgical NEC were missing anthropometric data. Weight $z$-scores did not differ by NEC/SIP status over time (interaction $p=0.2$ ), nor did the surgical NEC and SIP groups differ globally ( $p=0.77$, Fig. 3). However, values did differ over time $(p<0.001)$ for the entire cohort. Specifically, weight $z$-scores decreased from birth to discharge $(p<0.001)$, then increased from 4 to 8 months to $18-24$ months $(p=0.001)$. Height $z$-scores were found to differ by surgical NEC/SIP status over time (interaction $p=0.03$, Fig. 4. For surgical NEC patients, values significantly decreased from birth to discharge $(\mathrm{p}=$ 0.002 ), and remaining similar through 18-24 months. Among SIP patients, height $z$-scores declined from birth to discharge $(p<0.001)$ and again at the first follow-up visit $(p=0.044)$, then increased from 15-18 months to $18-24$ months $(p=0.03)$. Head circumference $z$-scores did not differ by NEC/SIP status over time (interaction $p=$ 0.13 ), nor did the surgical NEC and SIP groups differ globally ( $p=0.87$, Fig. 5). However, values did differ over time $(p=0.04)$ for the entire cohort. Head circumference zscores decreased from birth to discharge $(p=0.03)$, then remained similar through 18-24 months.

\section{Discussion}

In this study we found that height $z$-score differed between SIP and surgical NEC over time. Although both cohorts had significantly decreased height $z$-scores from birth to discharge, SIP infants had more catch up growth at 18-24 months of corrected age whereas the height z-scores of surgical NEC infants remained similar from discharge to 18-24 months of corrected age. We did not find a statistically significant difference in the weight outcomes in VLBW infants with surgical NEC or SIP. This is a surprising finding, given that infants with surgical NEC underwent bowel resection, had longer length of stay during their initial NICU hospitalization, and had greater use of PN and elemental formula compared to SIP infants. Although it
Table 2 Comparison of nutritional management of former very low birth weight infants with surgical necrotizing enterocolitis or spontaneous intestinal perforation.

\begin{tabular}{llll}
\hline & Surgical NEC, $n=14$ & SIP, $n=19$ & $p$ value \\
\hline Gastrostomy tube placement & $42.9 \%(6 / 14)$ & $15.8 \%(3 / 19)$ & 0.12 \\
Elemental formula at hospital discharge & $78.6 \%(11 / 14)$ & $21.1 \%(4 / 19)$ & 0.001 \\
Discharge home with parenteral nutrition & $21.4 \%(3 / 14)$ & $0 \%(0 / 19)$ & 0.07 \\
Elemental formula prescription at 15-18 months & $50 \%(6 / 12)$ & $0 \%(0 / 16)$ & 0.03 \\
corrected age & & & \\
Whole cow's milk at 15-18 months corrected age & $17 \%(2 / 12)$ & $50 \%(8 / 16)$ & 0.11 \\
\hline
\end{tabular}

Data are median (interquartile range) or percent (number). 


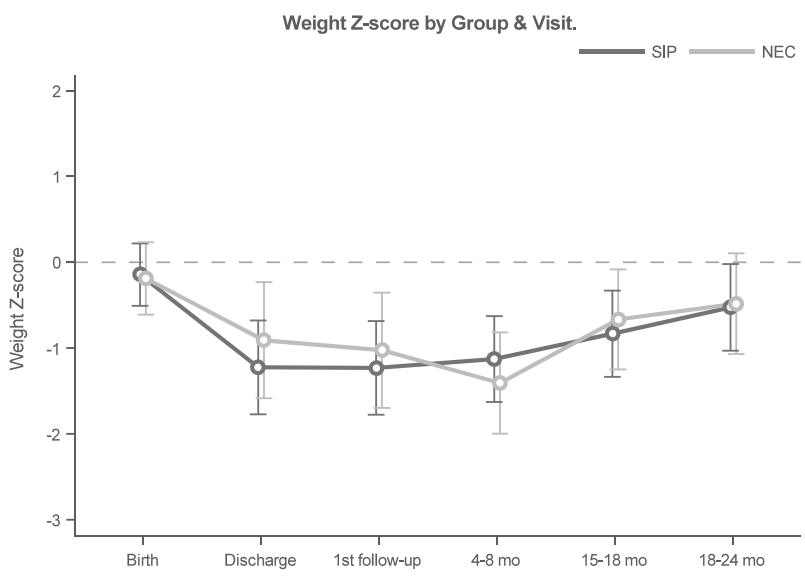

Fig. 3 Change in weight $z$-scores in very low birth weight infants with surgical necrotizing enterocolitis or spontaneous intestinal perforation from birth through hospital discharge and follow up at 24 months corrected age. Z-scores were calculated using the Fenton growth chart through 50 weeks corrected age and World Health Organization growth charts thereafter $[9,10]$. Linear mixed models were used to compare longitudinal weight outcomes by surgical necrotizing enterocolitis (NEC) and spontaneous intestinal perforation (SIP) groupings. Modeled means and 95\% confidence intervals were calculated by NEC/SIP status and study visit.

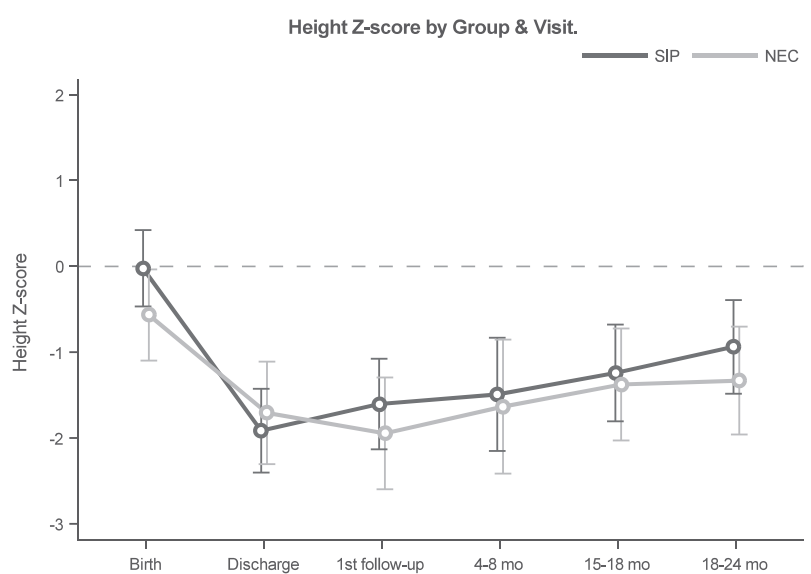

Fig. 4 Change in height $z$-scores in very low birth weight infants with surgical necrotizing enterocolitis or spontaneous intestinal perforation from birth through hospital discharge and follow up at 24 months corrected age. Z-scores were calculated using the Fenton growth chart through 50 weeks corrected age and World Health Organization growth charts thereafter [9, 10]. Linear mixed models were used to compare longitudinal height outcomes by surgical necrotizing enterocolitis (NEC) and spontaneous intestinal perforation (SIP) groupings. Modeled means and 95\% confidence intervals were calculated by NEC/SIP status and study visit.

was not a statistically significant difference, there was a trend towards higher numbers of subspecialist follow up appointments and additional hospitalizations in the group with surgical NEC. While many of these infants met the classic definitions of "extrauterine growth failure" or "postnatal growth failure" (weight $<10$ th percentile at

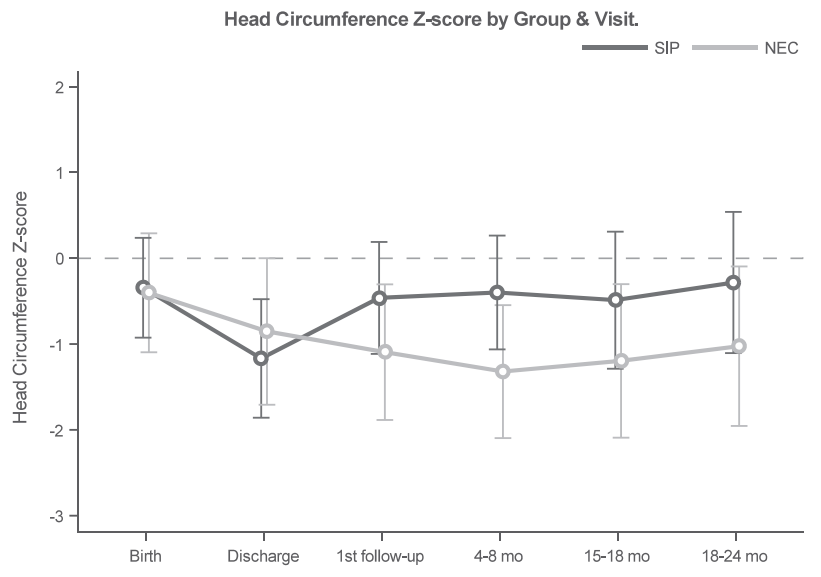

Fig. 5 Change in head circumference z-scores in very low birth weight infants with surgical necrotizing enterocolitis or spontaneous intestinal perforation from birth through hospital discharge and follow up at $\mathbf{2 4}$ months corrected age. Z-scores were calculated using the Fenton growth chart through 50 weeks corrected age and World Health Organization growth charts thereafter [9, 10]. Linear mixed models were used to compare longitudinal head circumference outcomes by surgical necrotizing enterocolitis (NEC) and spontaneous intestinal perforation (SIP) groupings. Modeled means and $95 \%$ confidence intervals were calculated by NEC/SIP status and study visit.

discharge), experts have recently suggested that other growth metrics are more important for predicting neurodevelopmental outcomes [12]. Postnatal head-sparing growth may be critical to optimize neurodevelopment, and this is important as infants with NEC are at risk for neurodevelopmental impairment [13-16].

These results are consistent with previous studies which demonstrated that preterm infants with surgical NEC have growth failure at 18-24 months [17-19]. Our study is novel in that it adds the details of nutritional management and reveals the growth differences in surgical NEC despite the prolonged duration of PN. Enteral autonomy is a consideration for classifying infants with intestinal failure, as this functionally defines whether infants are dependent on PN for survival. One definition of intestinal failure in infants is the need for PN for greater than 90 days $[4,20]$. Other experts use a cutoff duration of 60 days [21, 22]. Using either definition, a substantial proportion of infants with surgical NEC fulfilled the criteria for intestinal failure. This was true even though only $29 \%$ of these infants had short bowel syndrome per remaining intestinal length. In addition, $19.5 \%$ of the infants with medical NEC received $>60$ days of PN after diagnosis. Notably, most infants with medical NEC would be cared for in the regional Level 3 birth hospitals and only transferred to CCHMC for the need for subspecialist care, so this represents a biased population of infants with medical NEC. It is clear that the ability to achieve enteral autonomy is more complex than the length of bowel resected. 
One limitation of the study is the small sample size, thereby limiting statistical power to detect differences by surgical NEC and SIP groupings. The beginning of the epoch for inclusion was chosen to follow infants after institutional changes were made to the care of preterm infants, such as establishment of a donor milk protocol [23]. The endpoint was chosen so that all included infants would have growth outcome and follow up data through 24 months corrected age. Along with the low rates of NEC at our institution, this limited the potential sample size. Multicenter studies or networks might be able to generate a larger sample size, but they may have difficulty attaining the level of detail in management choices that we are able to describe. The results of this study may help inform future larger, multicenter studies.

The local protocols and management style of our institution and intestinal rehabilitation team may limit the generalizability of this study [24]. Use of elemental formula as a supplement to maternal milk following surgical NEC, for example, is part of our evidence-based approach to allow for bowel adaptation that may be center-specific [4, 24, 25]. Although patient and family variables are factored into a decision to place a gastrostomy tube or send an infant home with PN, there may be considerable center-based variations in care [21]. Our intestinal rehabilitation team considers continuous enteral feeding to be a critical element to assist in bowel remodeling for patients with intestinal failure, and gastrostomy tubes are placed to deliver continuous enteral feeding. Some of the foci of management strategies at our center include careful postoperative fluid and nutritional management, early and aggressive enteral feeding advancement, and use of PN to achieve goal macronutrient provision [25]. There is emerging literature on biomarkers to predict intestinal recovery [26]. Novel treatments such as teduglutide, a recombinant human glucagon-like peptide- 2 analog that promotes an intestinal adaptive response, may be the next strategy to achieve enteral autonomy in these patients [27].

\section{Conclusion}

Although the minority of infants with surgical NEC qualified as having short bowel syndrome using a remaining bowel length definition, the majority of the group had a duration of PN $>60$ days which is suggestive of physiological intestinal failure. VLBW infants with surgical NEC did not demonstrate catch-up linear growth as did infants with SIP by the 18-24 month corrected age follow up visit. Through 24 months corrected age, there was no difference in weight $\mathrm{z}$-scores between patients with surgical NEC and those with SIP. In addition to careful PN provision and use of elemental formula, novel therapeutics such as teduglutide might be the next area of research to help infants recovered from surgical NEC to achieve enteral autonomy.
Acknowledgements This project was supported in part by the National Center for Advancing Translational Sciences of the National Institutes of Health under Award Number 5UL1TR001425-03. The content is solely the responsibility of the authors and does not necessarily represent the official views of the NIH.

Author contributions KM designed the study, acquired, analyzed and interpreted the data, and drafted the manuscript; GG acquired and analyzed the data, and helped to write the manuscript; TJ analyzed and interpreted the data and critically revised the manuscript; AP acquired the data and critically revised the manuscript; JW, MH, and BP interpreted the data and critically revised the manuscript. All authors approve the final version to be published and agree to be accountable to all aspects of the work.

\section{Compliance with ethical standards}

Conflict of interest The authors declare that they have no conflict of interest.

Publisher's note Springer Nature remains neutral with regard to jurisdictional claims in published maps and institutional affiliations.

\section{References}

1. Engelstad HJ, Danko ME. Short Bowel syndrome in an infant. NeoReviews. 2020;21:e370-e382.

2. Ehrenkranz RA, Dusick AM, Vohr BR, Wright LL, Wrage LA, Poole WK. Growth in the neonatal intensive care unit influences neurodevelopmental and growth outcomes of extremely low birth weight infants. Pediatrics. 2006;117:1253-61.

3. Lin GC, Robinson DT, Olsen S, Reber KM, Moallem M, DiGeronimo $\mathrm{R}$, et al. Nutritional practices and growth in premature infants after surgical necrotizing enterocolitis. J Pediatr Gastroenterol Nutr. 2017;65:111-6.

4. Duggan CP, Jaksic T. Pediatric intestinal failure. N Engl J Med. 2017;377:666-75.

5. Goddard GR, McNelis K, Poindexter A, Jenkins T, Wessel J, Nathan AT, et al. Quality improvement efforts reduce incidence of surgical necrotizing enterocolitis and related deaths. Am J Perinatol. 2020 Jun 8. https://doi.org/10.1055/s-0040-1712967. Online ahead of print.

6. Bell MJ, Ternberg JL, Feigin RD, Keating JP, Marshall R, Barton L, et al. Neonatal necrotizing enterocolitis. Therapeutic decisions based upon clinical staging. Ann Surg. 1978;187:1-7.

7. Neu J. Necrotizing enterocolitis: the search for a unifying pathogenic theory leading to prevention. Pediatr Clin North Am. 1996;43:409-32.

8. Harris PA, Taylor R, Thielke R, Payne J, Gonzalez N, Conde JG. Research electronic data capture (REDCap) - a metadata-driven methodology and workflow process for providing translational research informatics support. J Biomed Inform. 2009;42:377-81.

9. Fenton TR, Kim JH. A systematic review and meta-analysis to revise the Fenton growth chart for preterm infants. BMC Pediatr. 2013;13:59.

10. WHO. Child growth standards based on length/height, weight and age. Acta Paediatr Suppl. 2006, 450:76-85.

11. Struijs MC, Diamond IR, de Silva N, Wales PW. Establishing norms for intestinal length in children. J Pediatr Surg. 2009;44:933-8.

12. Fenton TR, Cormack B, Goldberg D, Nasser R, Alshaikh B, Eliasziw M, et al. "Extrauterine growth restriction" and "postnatal 
growth failure" are misnomers for preterm infants. J Perinatol. 2020;40:704-14.

13. Guellec I, Lapillonne A, Marret S, Picaud JC, Mitanchez D, Charkaluk ML, et al. Effect of intra- and extrauterine growth on long-term neurologic outcomes of very preterm infants. J Pediatr. 2016;175:93-99.e91.

14. Meyers JM, Bann CM, Stoll BJ, D'Angio CT, Bell EF, Duncan $\mathrm{AF}$, et al. Neurodevelopmental outcomes in postnatal growthrestricted preterm infants with postnatal head-sparing. J Perinatol. 2016;36:1116-21.

15. Neubauer V, Griesmaier E, Pehböck-Walser N, Pupp-Peglow U, Kiechl-Kohlendorfer U. Poor postnatal head growth in very preterm infants is associated with impaired neurodevelopment outcome. Acta Paediatr. 2013;102:883-8.

16. Matei A, Montalva L, Goodbaum A, Lauriti G, Zani A. Neurodevelopmental impairment in necrotising enterocolitis survivors: systematic review and meta-analysis. Arch Dis Child Fetal Neonatal Ed. 2020;105:432-9.

17. Cole CR, Hansen NI, Higgins RD, Ziegler TR, Stoll BJ. Very low birth weight preterm infants with surgical short bowel syndrome: incidence, morbidity and mortality, and growth outcomes at 18 to 22 months. Pediatrics. 2008;122:e573-e582.

18. Hong CR, Fullerton BS, Mercier CE, Morrow KA, Edwards EM, Ferrelli KR, et al. Growth morbidity in extremely low birth weight survivors of necrotizing enterocolitis at discharge and two-year follow-up. J Pediatr Surg. 2018;53:1197-202.

19. Zozaya C, Shah J, Pierro A, Zani A, Synnes A, Lee S, et al. Neurodevelopmental and growth outcomes of extremely preterm infants with necrotizing enterocolitis or spontaneous intestinal perforation. J Pediatr Surg. 2020. May 15;S0022-3468(20)30326-2. https://doi.org/10.1016/j.jpedsurg.2020.05.013. Online ahead of print.

20. Sondheimer JM, Cadnapaphornchai M, Sontag M, Zerbe GO. Predicting the duration of dependence on parenteral nutrition after neonatal intestinal resection. J Pediatr. 1998;132:80-4.

21. Khan FA, Squires RH, Litman HJ, Balint J, Carter BA, Fisher JG, et al. Predictors of enteral autonomy in children with intestinal failure: a multicenter cohort study. J Pediatr. 2015;167: 29-34.e21.

22. Enman MA, Wilkinson LT, Meloni KB, Shroyer MC, Jackson TF, Aban I, et al. Key determinants for achieving enteral autonomy and reduced parenteral nutrition exposure in pediatric intestinal failure. J Parenter Enteral Nutr. 2019 Dec 16. https://doi.org/10. 1002/jpen.1754. Online ahead of print.

23. Nathan AT, Ward L, Schibler K, Moyer L, South A, Kaplan HC. A quality improvement initiative to reduce necrotizing enterocolitis across hospital systems. J Perinatol. 2018;38:742-50.

24. Jones $\mathrm{KD}$, Howarth LJ. Intestinal failure following necrotizing enterocolitis: A clinical approach. Early Hum Dev. 2016;97: 29-32.

25. Cole CR, Kocoshis SA. Nutrition management of infants with surgical short bowel syndrome and intestinal failure. Nutr Clin Pract. 2013;28:421-8.

26. Kuik SJ, Kalteren WS, Mebius MJ, Bos AF, Hulscher JBF, Kooi EMW. Predicting intestinal recovery after necrotizing enterocolitis in preterm infants. Pediatr Res. 2020;87:903-9.

27. Carter BA, Cohran VC, Cole CR, Corkins MR, Dimmitt RA, Duggan C, et al. Outcomes from a 12-week, open-label, multicenter clinical trial of teduglutide in pediatric short bowel syndrome. J Pediatr. 2017;181:102-111.e105. 